\title{
Estimation of Evoked Potentials Using Wavelet Transform Based Time-Frequency Adaptive Filtering
}

\author{
W. Q. Liu, W. Qiu, F.H.Y. Chan, F. K. Lam and P.W.F. Poon* \\ Department of Electrical and Electronic Engineering, University of Hong Kong, Hong Kong \\ * Department of Physiology, College of Medicine, National Cheng Kung University, Taiwan \\ E-mail:wqliu@eee.hku.hk,wqiu@eee.hku.hk, fhychan@eee.hku.hk
}

\begin{abstract}
In this paper a time-frequency domain adaptive filtering method is presented to estimate evoked potentials. Wavelet transform is used to represent the original responses in the time-frequency domain with a discrete set of wavelet coefficients. Each coefficient, which is related to the time extent and the frequency extent in the time-frequency plane, is processed by adaptive signal enhancer (ASE) to enhance signal components of the coefficient. The processed coefficients are then used to reconstruct the evoked potential signals with inverse wavelet transform. Visual evoked potentials (VEPs) from human subjects are estimated and good results are reached by this method.
\end{abstract}

Keywords: Wavelet transform, Adaptive filter bank, Time-frequency analysis, Evoked potentials.

\section{INTRODUCTION}

Evoked Potentials(EPs) are brain electrical activities resulting from sensory stimulation. EPs are very important in the diagnosis of the abnormalities of the nerve system. However the amplitude of the EP is only a few tenths of a microvolt, very much smaller than that of the background spontaneous EEG activity. Ensemble averaging (EA) is the most widely used method for obtaining the Eps [1]. However, this method ignores the fact that the EPs are also timevarying signals and the results of EA may introduce significant distortion and loss of information about the response variability from trial to trial. Digital filters are usually used to enhance the quality of EP signals. Adaptive filtering technique is one of the powerful methods for estimating the tome varying EP signals [1],[2]. In this paper, we present a new wavelet based time-frequency domain adaptive filtering method to estimate EP signals.

\section{METHODS}

\section{A. Wavelet Concept}

Recently, wavelet transform (WT) is widely used in the field of signal processing [3],[4],[5]. It has many advantages when compared with the traditional Short Time
Fourier Transform (STFT) and other time-frequency analysis methods such as Wigner-Ville distribution. The windows of WT can adaptively change both in time and frequency domains, so that it can zoom into details of the signals, making it very useful for time-frequency analysis of nonstationary signals. Besides, it also has a very important orthogonality feature. Every signal can be divided into two parts, which is orthogonal to each other. So the transformation is orthogonal.

The wavelet representation proposed by Daubechies [6] is used here. Let $\phi(x)$ be a scale function and $\psi(x)$ be the corresponding wavelet. Defining the coefficients of filters $\mathrm{H}$ and $\mathrm{G}$ as follows,

$$
\left\{\begin{array}{l}
h(n)=2^{-1 / 2} \int d x \phi\left(\frac{1}{2} x\right) \phi(x-n) \\
g(n)=2^{-1 / 2} \int d x \psi\left(\frac{1}{2} x\right) \psi(x-n)
\end{array}\right.
$$

Suppose the data of the $i$-th trial of evoked potential is $\left\{x_{i}(0), x_{i}(1) \cdots x_{i}(N-1)\right\}$, and for clarity, let it be $\left\{c_{0}^{0}, c_{1}^{0} \cdot, c_{N-1}^{0}\right\}$, so we have the decomposition formula:

$$
\left\{\begin{array}{l}
c_{k}^{j}=\sum_{n} h(n-2 k) c_{n}^{j-1} \\
d_{k}^{j}=\sum_{n} g(n-2 k) c_{n}^{j-1}
\end{array}\right.
$$

where $j$ indicates the scale, $\left\{c_{k}^{j}\right\}$ is the coarse signal, and $\left\{d_{k}^{j}\right\}$ is detailed signal. The corresponding reconstruction formula is:

$$
c_{n}^{j-1}=\sum_{k} h(n-2 k) c_{k}^{j}+\sum_{k} g(n-2 k) d_{k}^{j}
$$

In this paper, we use a total of 4 scales to do our analysis. So the signal in the transform domain can be expressed in the form $\left\{c_{0}^{4}, \ldots c_{N_{4}-1}^{4} ; d_{0}^{4}, \ldots d_{N_{4}-1}^{4} ; d_{0}^{3}, \ldots d_{N_{3}-1}^{3} ; \ldots d_{0}^{1}, \ldots d_{N_{1}-1}^{1}\right\}$ where $N_{j}=N / 2^{j}$. To simplify the notation in the subsequent adaptive filter implementation, it will be represented as $\left\{\alpha_{0}, \alpha_{1}, \cdots, \alpha_{N-1}\right\}$.

In dealing with the boundary problems, we use two approaches. By assuming the original signal is First, suppose 
the original signal is symmetric with respect to the first and the last sampled points, we have,

$$
\left\{\begin{array}{l}
c_{n}^{0}=c_{-n}^{0} \quad \text { if }-N<n<0 \\
c_{n}^{0}=c_{2 N-n-1}^{0} \quad \text { if } n \geq N
\end{array}\right.
$$

Another alternative is to assume the original signal is periodical, that is: $c_{k+N}^{0}=c_{k}^{0}$. In this case we can prove that

$$
\left\{\begin{array}{l}
c_{k+N / 2^{j}}^{j}=c_{k}^{j} \\
d_{k+N / 2^{j}}^{j}=d_{k}^{j}
\end{array}\right.
$$

then there is no need to extend the original signal. So the resulting algorithm is simple.

We use Daubechies' wavelets to process the signal. It is orthogonal with compact support. The order of regularity increases linearly with the support width. The evoked potential signal is rather regular. So we can choose the Daubechies' wavelet with 24 coefficients [6]. Fig.l shows the scale function and the corresponding wavelet. We can see that it has pretty high regularity.
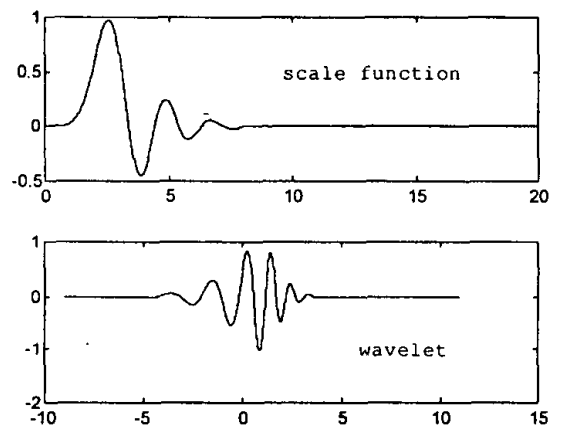

Figure 1: The Daubechies' wavelet with 24 coefficients

\section{B. Time-frequency domain adaptive filtering}

With discrete wavelet analysis, the time-frequency plane is divided into cells of constant which are related to each basic wavelet. Each cell of the plane is located around the time and frequency position of the related wavelet basis, and the height of the cell is proportional to the value of the related coefficient $\alpha_{i}(i=0,1, \cdots, N-1)$. Since the original data contain signal components and additive noise components, the coefficient $\alpha_{i}$ should be properly processed to get new coefficient $\alpha$; which could be used to reconstruct the signals with the noise components largely being reduced.

The time-locked EP signals are often expressed in the following additive signal model

$$
x_{i}(k)=s_{i}(k)+n_{i}(k) \quad k=0,1, \cdots, N-1
$$

where $x_{i}(k)$ is the $i$-th recorded response, while $s_{i}(k)$ denotes the $i$-th EP signal and $n_{i}(k)$ is noise which is usually considered to be uncorrelated with the response. Consider that the wavelet transformation is linear one, when the original responses are transformed into time-frequency domain by wavelet basis as equation (2), each coefficient $\alpha_{i}$ contains two components: $\alpha_{i}^{s}$ is related to the signal component of original response and $\alpha_{i}^{n}$ indicates the noise component. Therefore, the coefficients can be expressed as follows

$$
\alpha_{i}=\alpha_{i}^{*}+\alpha_{i}^{n} \quad(i=0,1, \cdots, N-1)
$$

If the coefficients can be processed by a such way that the noise components are largely reduced while the signal components are mainly kept, evoked potential signals of response can be obtained by inverse wavelet reconstruction using processed coefficients.

We use adaptive signal enhancer (ASE) to process the original coefficients. Fig.2 presents a version of ASE. There are two inputs in the ASE: the primary input contains a signal component $\alpha_{i}^{s_{i 1}}$ plus noise component $\alpha_{i}^{n_{0}}$, while the reference input contains a signal $\alpha_{i}^{s_{i}}$, related to but not necessarily having the same waveform as $\alpha_{i}^{s_{0}}$, and an additive noise $\alpha_{i}^{n_{1}}$. The noise $\alpha_{i}^{n_{0}}$ and $\alpha_{i}^{n_{1}}$ are assumed to be unrelated to each other and to both signals. The adaptive filter shown in Fig.1 iteratively adjusts its impulse response via an adaptive algorithm so that, after convergence, the difference between the filter output $y$ and the desired response $d$ is minimized. It can be shown the filter output is an optimal estimate of $\alpha_{i}^{s_{0}}$ in terms of MMSE, as discussed in [2].

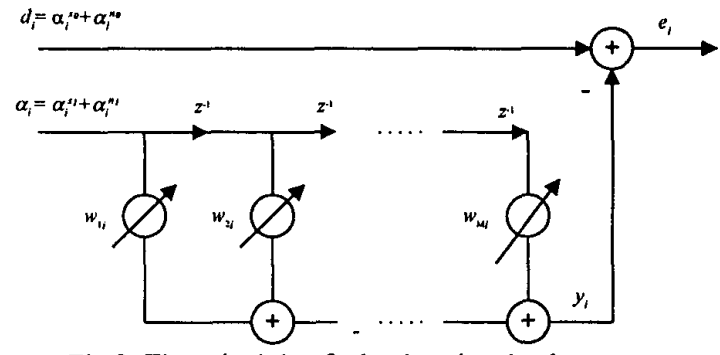

Fig.2 The principle of adaptive signal enhancer

It has been proved [2] that the improvement in SNR at the output of ASE is proportional to the SNR at the reference input. In order to obtain good results with less distortion and fast adaptation, it is desirable to reduce the noise components in the reference input. The reference input should be highly correlated with the signal. In fact, the average method provides a good reference input. LMS algorithm [7] is used for ASE. Since each cell of timefrequency domain is related to a particular time range and frequency band, each coefficient related to the cell should be processed singly by an ASE (as shown in Fig.3). 


\section{RESULTS}

We use the above new method to estimate visual evoked potential (VEP) of human beings. Fig. 4 shows two results of estimated VEP using wavelet-based time-frequency domain adaptive filtering and inverse wavelet reconstruction method and their corresponding original responses. With the help of the our method, it is possible to track the variation of EP signals. Fig.5 gives an isometric view of VEP traces. (Note that the peaks are inverted in Fig.5).

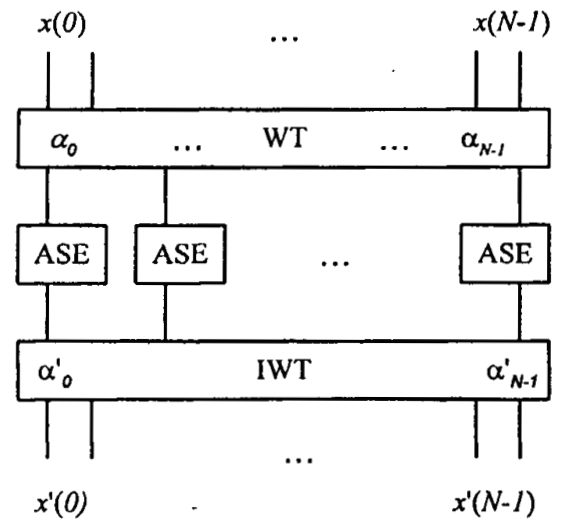

Fig.3 Wavelet based time-frequency domain adaptive filtering schematic diagram
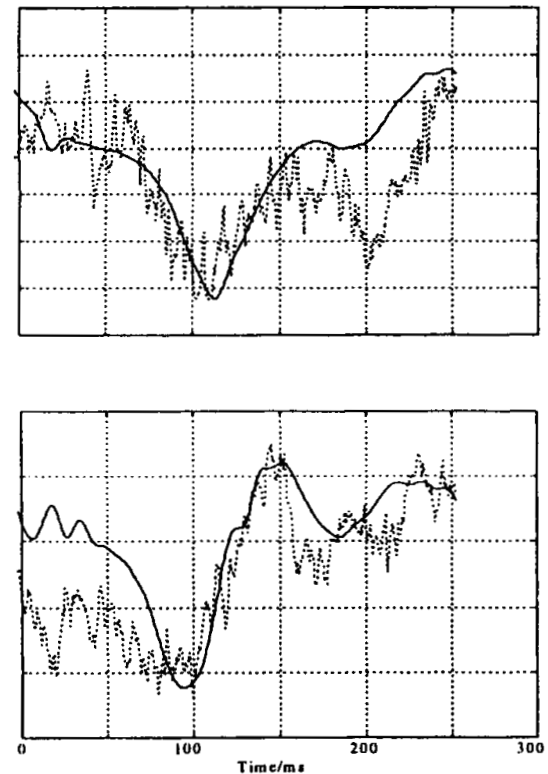

Fig.4 Two results of individual VEP estimation using wavelet based adaptive filtering and reconstruction technique. Solid lines are VEP estimation while dotted lines are corresponding original responses.

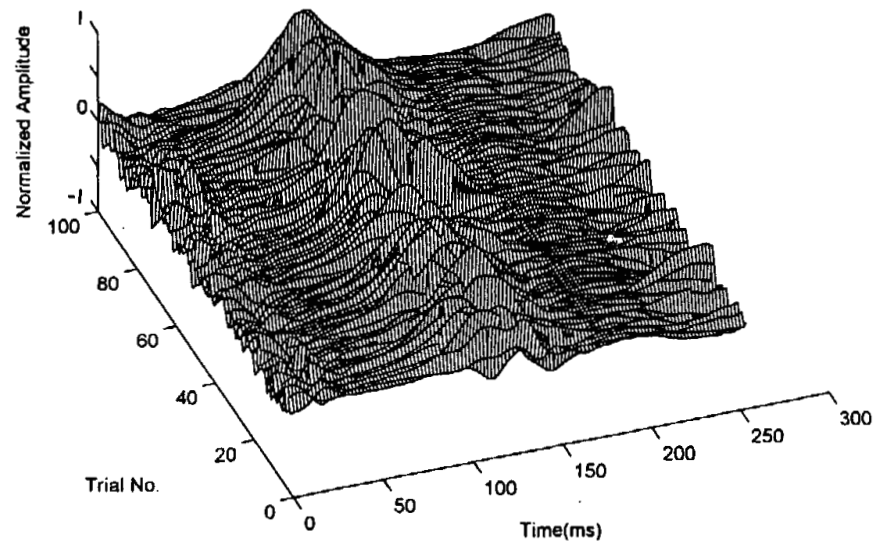

Fig.5 Isometric view of VEP trace across trials.

\section{CONCLUSION}

We have developed a wavelet based time-frequency domain adaptive filtering method to estimate EP signals. The original data is firstly transformed into time-frequency representation by wavelet method. The coefficients are then processed by ASE to enhance the signal components. Inverse wavelet transform is finally done using processed coefficients to get the EP estimation. Good VEP estimation has been achieved with this new method.

\section{REFERENCE}

[1] D. Regan, Human brain electrophysiology: evoked. potentials and evoked magnetic fields in science and medicine, Elsevier, New York, 1989.

[2] F.H.Y. Chan, F.K. Lam, P.W.F. Poon, W. Qiu, "Detection of brainstem auditory evoked potential by adaptive filtering", Med. \& Bio. Eng. \& Comput. Vol. 33, pp.69$75,1995$.

[3] S. G. Mallat, "A theory for multiresolution signal decomposition: the wavelet representation", IEEE Trans. Pattern Analysis and Machine Intelligence, Vol. 11 No.7, July, 1989.

[4] B. Oliver, B. Jorge, P. Jacques, "Time-frequency digital filtering based on an invertible wavelet transform: an application to evoked potentials", IEEE Trans. Biomed. Eng., Vol.41, No.1, pp.77-88, 1994.

[5] W. Q. Liu , F.H.Y. Chan, F.K. Lam and P.W.F. Poon, "Wavelet based methods for tracking the latency of evoked potentials", Proc. of 17th EMBS conference, pp. 1061-1062, 1995.

[6] I. Daubechies, "Orthonormal bases of compactly supported wavelets," Commun. Pure Appl. Math.. vol. XLI, pp.909-996,1988.

[7] B. Widrow, S.D. Stearns, Adaptive signal processing, Prentice-Hall, Inc. Englewood Cliffs, N.J., 1985. 